\title{
Finite-size effects on spin configurations in antiferromagnetically coupled multilayers
}

\author{
F. C. Nörtemann, ${ }^{*}$ R. L. Stamps, A. S. Carriço, ${ }^{\dagger}$ and R. E. Camley \\ Department of Physics, University of Colorado at Colorado Springs, Colorado Springs, Colorado 80933-7150
}

(Received 2 March 1992)

\begin{abstract}
Nonuniform canting states are found for multilayers of a finite size that are constructed from ferromagnetic films which are antiferromagnetically coupled. Our results are applicable to a wide variety of experimentally realizable systems such as $\mathrm{Fe} / \mathrm{Cr}$ and $\mathrm{Co} / \mathrm{Ru}$. We find that at low fields, a twist in the ground-state configuration of the spins reduces the net Zeeman energy and is energetically favorable to the uniform canted state which has previously been assumed by most authors. At higher fields, the character of this twist changes and eventually leads to a state that is fully aligned along the direction of the applied magnetic field. A numerical self-consistent mean-field model is used to examine the properties of these states and a variational method is developed in order to obtain analytic expressions for the lengths and magnitudes of these twists. The deviations from the uniform spin-flop state at both high and low fields can be quite large and involve the entire sample.
\end{abstract}

\section{INTRODUCTION}

Since the discovery of antiferromagnetic coupling between two Fe layers through an intervening nonmagnetic layer, ${ }^{1}$ there have been numerous experimental and theoretical investigations into this and similar systems. ${ }^{2}$ As a result, we now know that this phenomena can exist for a wide variety of spacer layers and magnetic films, and that there are a number of general features which seem to occur in all systems. ${ }^{3}$ Despite the large efforts which have gone toward understanding the mechanism for this antiferromagnetic coupling, the microscopic basis is still not entirely clear. ${ }^{4,5}$ Furthermore, there is an especially interesting associated effect which is of interest for applications. This is the appearance of huge magnetoresistances ${ }^{6-10}$ that depend on the relative orientation of the spins from layer to layer.

Theories for magnetoresistance, magnetic susceptibilities, and spin-wave dynamics in these antiferromagnetically coupled systems are extremely dependent on the configuration of the spins, but up to now theoretical works have supposed only very simple ground states. Surprisingly, very little has been done on the true configuration of the ground state for finite multilayers composed of antiferromagnetically coupled ferromagnets. We show in this paper that the low-field configurations are not at all simple and can be dramatically different from previously assumed configurations.

The states we will discuss here are reminiscent of surface phase transitions where the outermost spins are twisted away from the bulk equilibrium directions. ${ }^{11}$ An interesting and peculiar feature of the configurations studied here, however, is that at both low and high fields the twist involves spins throughout the entire multilayer.

The system we consider is composed of ferromagnetic films which are weakly coupled with one another antiferromagnetically. The spins are assumed to lie parallel to the film planes, and so there are no static demagnetizing fields. We also assume that the individual magnetic films are very thin, consisting of no more than five or ten atomic layers. This means that the spins within an individual film remain parallel to one another because of a strong intralayer-exchange coupling. The systems are at finite temperatures, but well below the Curie temperature of the ferromagnet, so that a semiclassical treatment is appropriate. For simplicity, we ignore anisotropies, so that the only relevant energies are the interface-exchange coupling between films and the Zeeman energy due to the interaction of an external applied magnetic field and the net magnetic moment in each film.

Before discussing the surface-twist state investigated in this paper, we first review the calculation for the spin configuration in an infinitely extended, bulk, layered structure. To do this, we examine the Zeeman and exchange energies of $N$ antiferromagnetically coupled films. One possible simple configuration is a spin-flop state shown in Fig. 1(a). In this figure the magnetization of each film is represented by an arrow. Here the spins in adjacent layers are canted away from one another by an angle $2 \alpha_{0}$. The energy of this configuration is

$$
E_{b}=-J(N-1) \cos \left(2 \alpha_{0}\right)-H_{0} M N \cos \left(\alpha_{0}\right),
$$

where $\alpha_{0}$ is the angle between the net magnetic moment $M$ of a film and the external field $H$. The interfaceexchange energy is denoted $J$ and is negative for antiferromagnetic coupling. The equilibrium configuration for the layers is defined by the angle $\alpha_{0}$ that minimizes Eq. (1). This angle is appropriate to a spin far from the ends of the multilayer stack and can be obtained by letting $N$ be very large in Eq. (1). This is given by

$$
\cos \left(\alpha_{0}\right)=-\frac{H_{0} M}{4 J} \text {. }
$$

The simple spin-flop state described above is, however, not a stable state for a finite multilayer and, as we will see, is a reasonable approximation to the true state only in certain limits. 
The surface-twist structure in finite multilayers is allowed because of the reduced symmetry of the end spins; i.e., the end layers of a finite multilayer experience half the exchange coupling of the spins in the middle of the multilayer. This means that it costs less exchange energy to twist an end layer in the direction of the applied field than to twist a bulk layer into the field direction. Thus if a twist is energetically favorable, it should begin at a free end.

Examples of configurations for finite structures which have lower energies than the uniform canted state of Fig. 1(a) are shown in Figs. 1(b) and 1(c). We note that the structure in Fig. 1(b) is quite complex. While the outermost spins are twisted into the direction of the field, the second layers of spins are actually turned farther away from the field than they would be in the bulk configuration. This alternation, one spin pointing closer to the field and the next farther away, continues as one progresses into the bulk, but the amplitude of the deviation decreases with increasing distance from the surface. The main difference between the configuration in Fig. 1(b) and that of Fig. 1(c) is that there is no alternation in

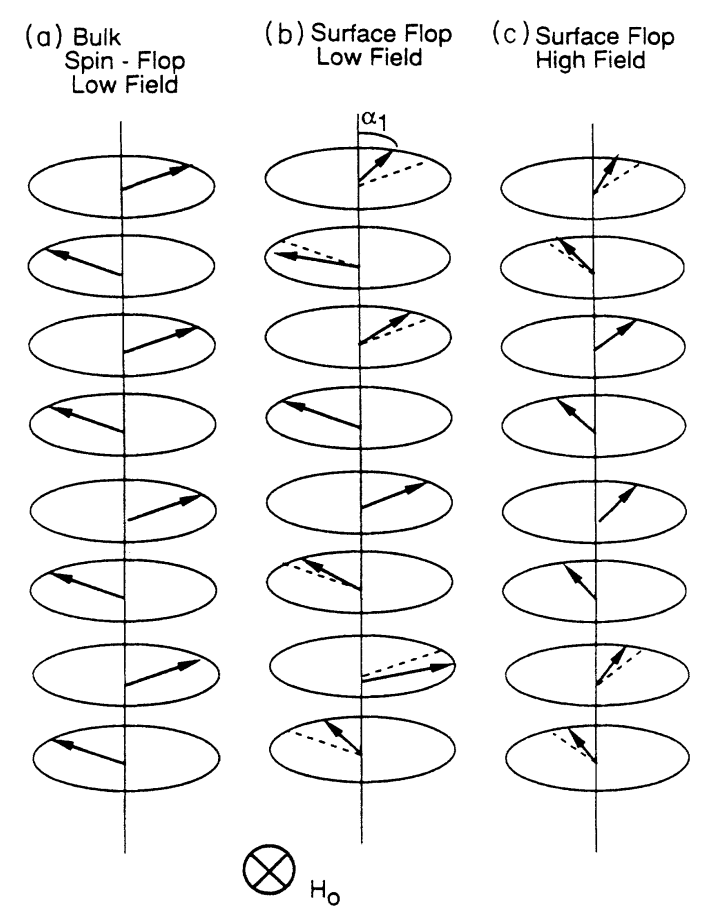

FIG. 1. Examples of possible configurations for a multilayer composed of antiferromagnetically coupled ferromagnetic thin films. The magnetization of each thin film $n$ lies in the plane of the film, is represented by an arrow, and makes an angle $\alpha_{n}$ with the external field $H_{0}$. In (a) the "bulk" state is shown where $\alpha_{n}$ is the same for all films. (b) and (c) show configurations for a finite multilayer, with the upper part of the diagram being the top surface of the multilayer and the lower part shows the bottom surface of the multilayer. The dashed lines in (b) and (c) show the "bulk" configuration for these cases. Note that for (b) the outermost spins are turned into the direction of the field, but that the next layer has the spins turned away from the field when compared with the bulk configuration. the high-field configuration shown in Fig. 1(c).

It should be mentioned that the nonuniform canted states discussed here are very different in nature from canted states within a ferromagnetic film. For example, in a ferromagnet there exist domain walls where the spins rotate from the magnetization direction. The width of a domain wall is governed by the competition between the exchange and anisotropy energies. The exchange energy can be minimized by spreading the canting out over many layers of spins and thus favors a very extended wall. In contrast, the anisotropy energy is minimized when spins point in the easy direction and this favors a narrow wall. In our problem we also have competition between two energies, but here it is the exchange and Zeeman energies. However, in the multilayer structure considered here, we have the additional freedom of having two sublattices. This allows new kinds of states to emerge when trying to minimize the total energy. The alternating spin configuration seen in Fig. 1(b), for example, has the exchange energy between layers of spins alternately increase and then decrease as we penetrate into the material from the surface. This extra degree of freedom allows the width of the nonuniform canting region to vary in an interesting manner as a function of applied field. At low fields the nonuniform region is very large; for moderate fields it is quite small, and then for larger fields the width of the nonuniform region is again quite large.

These nonuniform canted states will be discussed in detail in the following sections. In the next section, we use a numerical model that searches for the configuration that minimizes the total energy in a mean-field approximation. In Sec. III we present a variational technique which assumes certain ground states and yields analytic expressions for the magnitude and length of the twists.

\section{SELF-CONSISTENT MEAN-FIELD RESULTS}

The details of this method have been presented elsewhere, ${ }^{12}$ and so we only briefly comment on the calculational technique. The idea is simply to calculate the effective field acting on an individual spin due to its neighbors and then to rotate the spin in such a way as to minimize its energy. This is done for each spin in the structure and repeated until a stable configuration appears. When more than one stable configuration is possible, the configuration with the lowest energy is assumed to be the equilibrium configuration.

In the numerical model, each film consists of two or more layers of ferromagnetically coupled spins. The outermost spins of each film are coupled antiferromagnetically to the outermost spins of the neighboring films. As discussed above, the films are very thin and the intralayer exchange interactions are strong enough so that the spins within a film remain parallel to one another. In order to keep our results as general as possible and independent of the particular magnetic material used in the layers, we use the following reduced units. We define the parameter $h$ as

$$
h=\frac{H_{0} M}{|J|},
$$



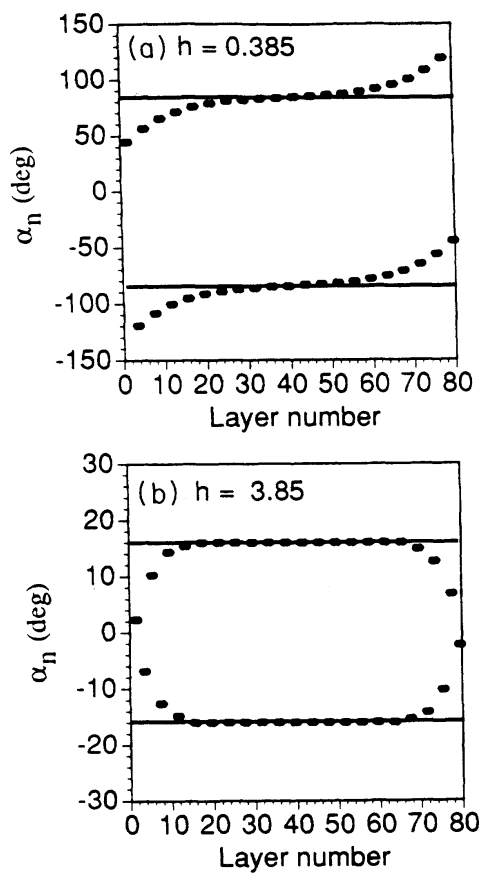

FIG. 2. Angle $\alpha_{n}$ is shown as a function of $n$ for (a) a lowfield case and (b) a high-field case. The multilayer contains 40 films with 2 layers of spins per film. The solid horizontal lines show the canting angles for an infinitely extended multilayer.

where $M$ is the net magnetic moment for the film and is given by $M=N_{L} M_{L}$, where $N_{L}$ is the number of atomic layers across the film and $M_{L}$ is the magnetic moment of an individual layer. At $h=4$, Eq. (2) implies that the spins are aligned in the direction of the applied field.

In Fig. 2 we show how the angle $\alpha_{n}$ varies across a 40film multilayer stack. Each film is two atomic layers thick in this example. The applied field in Fig. 2(a) is $h=0.385$. This corresponds to a bulk canting angle $\alpha_{0}=84.5^{\circ}$ from Eq. (2) and is shown by the solid lines. The configuration shown in Fig. 2(a) is depicted schematically in Fig. 1(b). For this configuration the deviation of the angle $\alpha$ from the bulk spin-flop angle alternates in sign from film to film. Note that this configuration is antisymmetric across the midplane of the sample. Thus, if the surface twist is created by twisting the spins near the upper surface to the right, the spins near the lower surface are twisted to the left. This allows the net magnetization to always point in the direction of the applied field. As can also be seen, the angle between spins within each film is zero. This is because the ferromagnetic coupling between spins within a layer is much stronger than the antiferromagnetic coupling between films.

A very interesting feature is that at low fields the twist involves the entire sample and results in significant deviations from the bulk spin-flop state. In the example seen in Fig. 2(a), the angular position of the spins varies from about $+45^{\circ}$ to $+84.5^{\circ}$ and from $-84.5^{\circ}$ to $-120^{\circ}$ near the upper surface. The penetration length of the surface twist is also quite large, extending about 10 unit cells into the bulk from each end. As the field is increased, the twist initially becomes more localized to the ends.

If the magnitude of the field is increased even further, the character of the spin configuration changes. An example is shown in Fig. 2(b), where $h=3.85$. Here $\alpha_{0}=15.7^{\circ}$, and we see that a new configuration has appeared which consists of a turning of each magnetization into the direction of the applied field such that $\left|\alpha_{n}\right|<\left|\alpha_{0}\right|$. For $h=3.85$ only the first six films at each end are turned toward the field. As the field is increased, the number of films with $\left|\alpha_{n}\right|<\left|\alpha_{0}\right|$ also increases. This continues until all of the spins point in the direction of the field. This type of nonuniform canting is shown schematically in Fig. 1(c).

The configurations shown in Figs. 1(b) and 1(c) are energetically more favorable than the uniformly canted configuration of Fig. 1(a) because the net magnetization in the direction of the field for the configurations of Figs. 1 (b) and 1(c) is increased as a result of nonuniform canting. This is clearly seen in Fig. 3, where the average magnetization in the direction of the applied field is shown as a function of applied-field strength. Three different cases are shown for comparison: A system of four films, a system of ten films, and the uniformly canted configuration of Fig. 1(a). The average deviation from the bulk canting angle $\alpha_{0}$ decreases as the number of films in the stack increases, and as a result, the average magnetization for larger multilayers approaches the bulk value. In all cases the magnetization of each layer is completely aligned along the applied field for $h \geq 4$.

The spins in the outermost layers of the multilayer are the easiest to turn into the applied-field direction and therefore have the largest deviations from the bulk orientation. It is therefore reasonable to expect that it should be possible to increase the magnitude of the deviations from the bulk state by decreasing the total length of the multilayer stack. This is illustrated in Fig. 4, where the average magnetization of a multilayer stack is shown as a function of the total number of layers composing the stack. The applied field is constant at $h=1.541$. We see here that the magnetization increases as the total number of films decreases, indicating that the net deviation of the

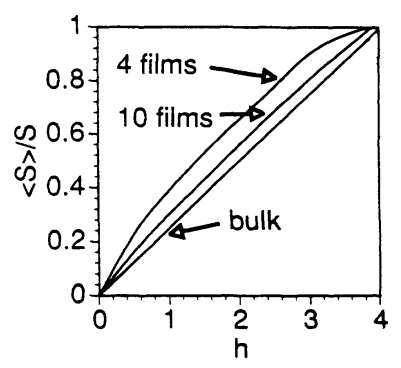

FIG. 3. Average magnetization for a multilayer is shown as a function of applied field for a four-film multilayer, a ten-film multilayer, and an infinitely extended multilayer ("bulk" case). In all cases the spins are aligned with the field when $h=4$. Note that the deviation from the bulk case is largest for the multilayer with the least number of layers. 


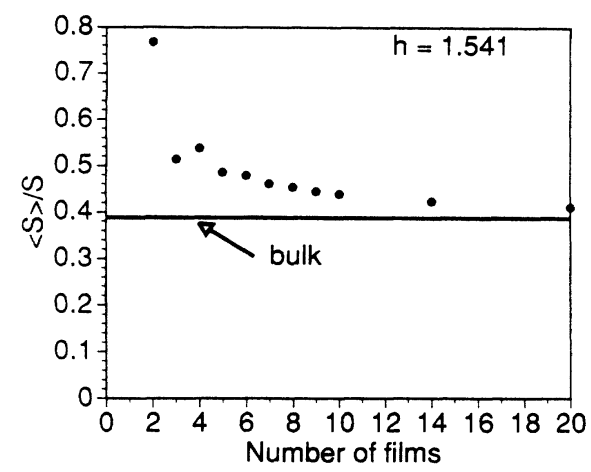

FIG. 4. Average magnetization for a multilayer as a function of the total number of layers. The applied field is $h=1.541$. The nonuniform twist states exist because of the reduced symmetry of the end films. As a consequence, the net deviation from the bulk state increases with decreasing number of layers, which leads to an increasing magnetization. Note the difference between even numbers of films and odd numbers of films.

canting angle from its bulk value $\alpha_{0}$ also increases as the total number of films decreases.

In Fig. 3 we saw how the magnetization as a function of field differed from the bulk behavior due to finite-size effects on the multilayer stack. Both of the examples shown in Fig. 3 were for multilayers with an even number of films. In Fig. 5 we again show the magnetization as a function of field strength, but for multilayers consisting of an odd number of films. The behavior for these cases is quite different from the even-numbered systems for low fields. What happens is that an odd number of layers gives the system a net magnetic moment in zero applied field. This makes the system relatively stable to small applied fields, since the net magnetization prefers to remain aligned in the field direction rather than go into a canted state. As the total number of films is increased, the average magnetization becomes proportionally smaller and the system goes into a canted state at much smaller fields. This feature has already been observed in recent experiments. ${ }^{13}$ The low-field canted state for an odd number of films is also different from that for an even number of

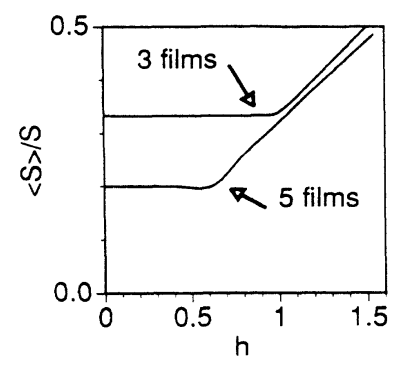

FIG. 5. Average magnetization of a multilayer with an odd number of films is shown as a function of applied field. The multilayer then has a net magnetization that prevents a canted state at low fields.

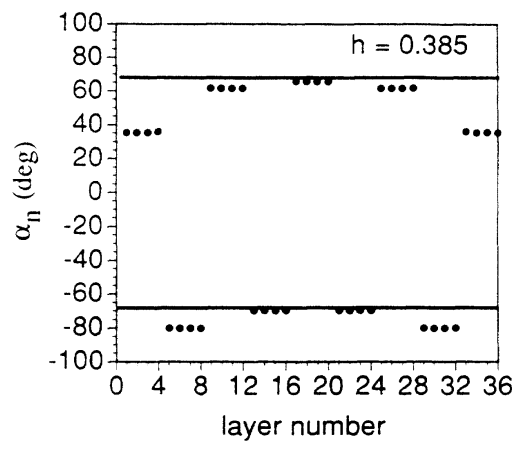

FIG. 6. Angle $\alpha_{n}$ is shown as a function of $n$ for a multilayer with an odd number of films. In this example the total number of layers is 9. For odd-numbered cases, the angle is symmetric about the center of the multilayer.

films. In contrast to the twist shown Fig. 2, the twist in the structure with an odd number of films is symmetric with respect to the middle of the stack. This can be seen in Fig. 6, where we show the canting angle $a$ as a function of position for a stack of nine films.

Finally, we note that a hysteresis loop would not appear without the presence of anisotropies if we were to continue on and reverse the direction of the applied field. The presence of anisotropies, however, would lead to hysteresis curves similar to those found in the experiment. ${ }^{13}$ Such hysteresis effects for systems a small number of layers have been treated theoretically earlier. ${ }^{14}$

\section{VARIATIONAL CALCULATION}

We now discuss in more detail the unusual configurations described in the last section. Our goal is to use a simple variational technique to estimate the magnitude of the deviations and also the number of layers involved. In Fig. 1(b), a possible configuration is shown for a finite multilayer in a small applied field. As a crude approximation, we represent one end of this configuration by defining a trial function for $\alpha$ as

$$
\alpha_{n}=\alpha_{0}+(-1)^{n} B(L-n) \text {, }
$$

where $n$ denotes the layer number, $\alpha_{n}$ is the angle between the spins of layer $n$ and the applied field, $B$ is the amount of twist between layers, and $L$ is the length of the twist. For simplicity, we limit ourselves to a semi-infinite superlattice so that there is only a twist at one end. We note that the $(-1)^{n}$ term introduces the alternation in angular position discussed earlier. This trial function will then be used in the expression for the total energy of the multilayer, and values for $B$ and $L$ will be sought such as to minimize the energy.

The difference in energy between the uniform bulk state and the energy of a state with surface twist extending $L$ layers is given by 


$$
\begin{aligned}
E= & L\left[J \cos \left(2 \alpha_{0}\right)+H_{0} M \cos \left(\alpha_{0}\right)\right]-J \sum_{n=1}^{L}\left[\cos \left(2 \alpha_{0}\right) \cos (B)-(-1)^{n} \sin \left(2 \alpha_{0}\right) \sin (B)\right] \\
& -H_{0} M \sum_{n=1}^{L}\left\{\cos \left(\alpha_{0}\right) \cos [B(L-n)]-(-1)^{n} \sin \left(\alpha_{0}\right) \sin [B(L-n)]\right\}
\end{aligned}
$$

The sums in Eq. (5) can be explicitly evaluated, with the result

$$
\begin{aligned}
E= & L\left[J \cos \left(2 \alpha_{0}\right)+H_{0} M \cos \left(\alpha_{0}\right)\right]-J L \cos \left(2 \alpha_{0}\right) \cos (B) \\
& -H_{0} M \cos \left(\alpha_{0}\right)\left\{\frac{\cos [(L / 2) B] \sin \{[(L+1) / 2] B\}}{\sin \left(\frac{1}{2} B\right)}-\cos (L B)\right\} \\
& +H_{0} M \sin \left(\alpha_{0}\right)\left\{\frac{\sin [(L / 2) B+(L / 2) \pi] \sin \{[(L+1) / 2](\pi-B)\}}{\cos \left(\frac{1}{2} B\right)}-\sin (L B)\right\} .
\end{aligned}
$$

We seek conditions on $B$ and $L$ such that this energy is minimized. The linear approximation for Eq. (4) is probably only good for cases where $L \gg 1$ and $B \ll 1$. It is therefore reasonable to perform an expansion in $B$. Assuming that both $B \ll 1$ and $B L \ll 1$ and expanding Eq. (6) to second order in $B$, we find

$$
E=-J \cos \left(2 \alpha_{0}\right)\left[L-\frac{L}{2} B^{2}+\frac{B^{2}}{2}\right]-J B \sin \left(2 \alpha_{0}\right)+H_{0} M\left\{\frac{B L}{2} \sin \alpha_{0}+L\left[1-B^{2}\left[\frac{1}{6} L^{2}-\frac{1}{4} L+\frac{1}{12}\right]\right] \cos \left(\alpha_{0}\right)\right\} .
$$

It is now a simple matter to minimize $E$ with respect to $L$ and $B$. This results in two equations which can be solved for $L$ and $B$ in terms of $\alpha_{0}$. It is convenient to write the resulting equations entirely as functions of $H_{0}, J$, and $M$ using $\alpha_{0}$ from Eq. (2). For convenience, we write the results in terms of the unitless parameter $h$ defined in Eq. (3), which measures the relative strength of the Zeeman energy to the exchange energy. The expressions for $L$ and $B$ then become

$$
L=1+\left(\frac{12}{h^{2}}-1\right)^{1 / 2}
$$

and

$$
B=\frac{12 h\left(1-h^{2} / 16\right)^{1 / 2}}{h^{2} L(2 L-1)-3 h^{2}+24} .
$$

Note that real roots for $L$ occur only for small $h$, which is consistent with our assumption that the trial function given by Eq. (4) represents the low-field configuration. As $h$ increases, $L$ decreases until $L=1$ at approximately $h=3.464$, which is below the field $h=4$ where the angle between spins is zero. Also, we see that $B$ decreases with increasing $h$.

Next, we calculate $L$ and $B$ for higher fields. In this case we use a trial function that describes the configuration shown in Fig. 1(c). The trial function is now

$$
\alpha_{n}=\alpha_{0}-B(L-n) \text {. }
$$

Following the same arguments as before, we eventually arrive at the following expressions analogous to Eqs. (8) and (9) above. These equations have a much more complicated dependence on $h$ and $\alpha_{0}$ than in the low-field case:

$$
\begin{aligned}
& L^{3}\left[\frac{h^{2}}{24} \sin \left(2 \alpha_{0}\right)-\frac{h}{3} \cos \left(2 \alpha_{0}\right) \sin \alpha_{0}\right]+L^{2}\left[\sin \left(4 \alpha_{0}\right)-\frac{h}{2} \cos \alpha_{0} \sin \left(4 \alpha_{0}\right)\right] \\
& +L\left[-\sin \left(4 \alpha_{0}\right)+\frac{h}{2}\left[\cos \alpha_{0} \sin \left(2 \alpha_{0}\right)+\frac{11}{6} \sin \alpha_{0} \cos \left(2 \alpha_{0}\right)\right]-\frac{h^{2}}{48} \sin \left(2 \alpha_{0}\right)-\frac{h}{3} \cos \left(2 \alpha_{0}\right) \sin \alpha_{0}\right] \\
& +\frac{11}{12} \sin \left(4 \alpha_{0}\right)-\frac{h}{2}\left[\frac{1}{6} \cos \alpha_{0} \sin \left(2 \alpha_{0}\right)+\sin \alpha_{0} \cos \left(2 \alpha_{0}\right)\right]=0
\end{aligned}
$$

and

$$
B=\frac{\sin \left(2 \alpha_{0}\right)-(h L / 2) \sin \alpha_{0}}{\left[\frac{11}{3} \cos \left(2 \alpha_{0}\right)-(h / 6) \cos \alpha_{0}\right] L-\left[(h / 2) \cos \alpha_{0}-4 \cos \left(2 \alpha_{0}\right)\right] L^{2}+\left[\frac{4}{3} \cos \left(2 \alpha_{0}\right)-(h / 3) \cos \alpha_{0}\right] L^{3}-\cos \alpha_{0}} .
$$


We want to compare the results of the variational method with the results from the numerical model. It is important to note, however, that the numerical calculation produces a configuration without the assumptions implicit in our variational trial function, i.e., the linear dependence of $\alpha$ on $B$ and $L$. While the initial angle from the numerical calculation can be directly compared to $B L$, it is necessary to somehow define a length for the numerical calculation that is comparable to $L$. We do this by comparing $L$ to the length in the numerical model where the total angular deviation $\alpha_{L}-\alpha_{0}$ is reduced to $90 \%$ of its initial value.

In Fig. 7 the length $L$ is plotted as a function of the reduced field $h$, and in Fig. 8 the angle of the first layer spins, $\alpha_{1}$, is shown as a function of $h$. The solid lines are the results of our low- and high-field variational treatments. The circles are taken from the numerical calculation described in the last section. In the figures we see that the variational model gives the same general trends as in the numerical model. The best agreement in both cases is found at higher fields, where $\alpha_{1}$ is the smallest.

A curious feature is the behavior of $L$ as a function of applied field. For low fields, $L$ is at first very large and then decreases with increasing field. At low fields the Zeeman energy is reduced by forming a configuration such as that shown in Fig. 1(b) that increases the net magnetization in the field direction. The cost of this configuration in exchange energy is reduced by spreading it across the entire stack of films. As the field is increased, the deviations occur only for films near the ends of the stack. At some moderate field strength, the old configuration disappears and a configuration of the type shown in Fig. 1(c) becomes energetically favorable. This new configuration reduces the Zeeman energy by simply turning the magnetization of the outermost films into the field direction. The number of films which participate in this turning increases with increasing field, to finally result in the magnetization of all the films being in the field direction when $h=4$. For this configuration, then, $L$ increases with $h$.

The results of Fig. 8 suggest a straightforward possibility for experimental verification of the surface-twist

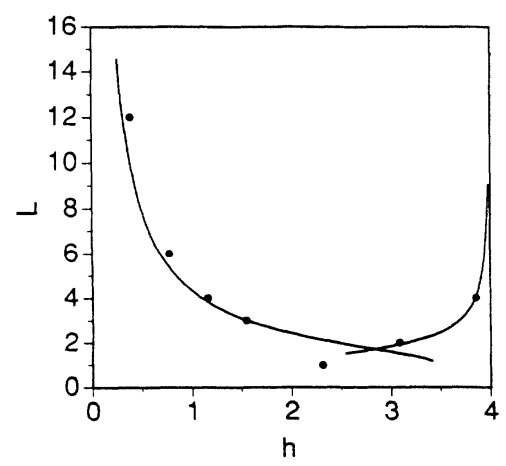

FIG. 7. Results of the variational calculation for the length $L$ as a function of applied field. The solid lines represent the results of the variational calculations, and the dots are the equivalent results from the numerical calculation. Note the good agreement at high fields and large $L$.

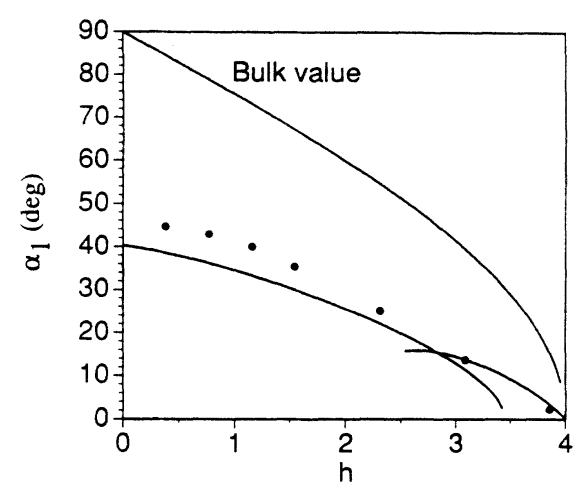

FIG. 8. First angle $\alpha_{1}$ is shown as a function of applied field. The solid lines are the results from the variational calculations and the dots are the results from the numerical calculation. The agreement between the two methods improves with increasing field as the magnitude of $\alpha_{1}$ decreases.

structure. Figure 8 shows that the surface canting angle $\alpha_{1}$ is always considerably smaller than the bulk canting angle $\alpha_{0}$. If $L$ is not too large, a measurement of the net magnetic moment will give a good approximation for the bulk canting angle. However, a measurement of the magneto-optical Kerr effect (MOKE), which is sensitive to the magnetic moment near the surface, should give a good account of the surface canting angle. Thus, if these two measurements are compared, one should be able to see the difference between the bulk and surface behavior.

\section{CONCLUSIONS}

We have examined the ground-state configuration of multilayers constructed from antiferromagnetically coupled ferromagnetic films. In the presence of an external magnetic field, finite multilayers display a rich variety of ground-state configurations that are fundamentally different in character from the ground-state configurations of an infinitely extended multilayer. At some applied-field strengths, these finite-size effects are localized to the films at the ends of the multilayer stack, while for other applied-field strengths the entire multilayer is affected.

There are a number of consequences which we have not yet explored. One of the most interesting questions is the effect of the states discussed here on the longwavelength spin-wave spectrum of the multilayer. The magnitude of effects discussed here can be quite large for easily accessible field strengths and may have a significant effect on the surface spin-wave modes which are easily observable through techniques such as Brillouin light scattering. A second interesting question relates to applications of the giant magnetoresistance effect found in some of these multilayer structures. Magnetoresistive sensors often operate at low fields and in a range where the resistivity changes linearly with applied field. It remains to be seen if the nonuniform canted structure discussed in this paper will help or hinder in obtaining these operating characteristics. 


\section{ACKNOWLEDGMENTS}

This work was supported by the U.S. Army Research Office through Grant No. DAAL0391-G-0229. One of us (F.C.N.) thanks Professor G. Güntherodt and Dr. B. Hil- lebrands of the 2. Physikalisches Institut at the RWTH Aachen for supporting participation in this work. A.S.C. gratefully acknowledges the Fullbright Foundation and the CNPq for support. R.E.C. would also like to thank G. Felcher for a stimulating conversation.
*Permanent address at 2. Physikalisches Institut, RWTH Aachen, Aachen, 5100 Germany.

†On leave from Departamento de Fisica, Universidade Federal do Rio Grande do Norte, 59072 Natal/RN Brasil.

${ }^{1}$ P. Grünberg, R. Schreiber, Y. Pang, M. B. Brodsky, and H. Sowers, Phys. Rev. Lett. 57, 2442 (1986).

${ }^{2}$ S. S. P. Parkin, N. Moore, and K. P. Roche, Phys. Rev. Lett. 64, 2304 (1990).

${ }^{3}$ S. S. P. Parkin, Phys. Rev. Lett. 67, 3598 (1991).

${ }^{4}$ D. M. Edwards, J. Mathon, R. B. Muniz, and M. S. Phan, Phys. Rev. Lett. 67, 493 (1991).

${ }^{5}$ Y. Wang, P. M. Levy, and J. L. Fry, Phys. Rev. Lett. 65, 2732 (1990).

${ }^{6}$ M. N. Baibich, J. M. Broto, A. Fert, F. Nguyen Van Dau, F. Petroff, P. Etienne, G. Creuzet, A. Friederich, and J. Chaze- las, Phys. Rev. Lett. 61, 2472 (1988).

${ }^{7}$ G. Binasch, P. Grünberg, F. Saurenbach, and W. Zinn, Phys. Rev. B 39, 4828 (1989).

${ }^{8}$ R. E. Camley and J. Barnas, Phys. Rev. Lett. 63, 664 (1989).

${ }^{9}$ P. M. Levy, S. Zhang, and A. Fert, Phys. Rev. Lett. 65, 1643 (1990).

10J. J. Krebs, P. Lubitz, A. Chaiken, and G. A. Printz, Phys. Rev. Lett. 63, 1645 (1989).

${ }^{11}$ J. G. LePage and R. E. Camley, Phys. Rev. Lett. 65, 1152 (1990).

${ }^{12}$ R. E. Camley and D. R. Tilley, Phys. Rev. B 37, 3413 (1988).

${ }^{13}$ S. S. P. Parkin, A. Mansour, and G. P. Felcher, Appl. Phys. Lett. 58, 1473 (1991).

${ }^{14}$ B. Dieny, J. P. Gavigan, and J. P. Rebouillet, J. Phys. Consens. Matter 2, 159 (1990). 